\title{
HIV infection and sex in sex-on-premises venues are associated with a higher risk of syphilis reinfection among men who have sex with men
}

\author{
Maciej Pastuszczak ${ }^{1}$ Monika Bociąga-Jasik², Marek Sitko², Anna Wojas-Pelc \\ 'Department of Dermatology, School of Medicine, Jagiellonian University, Krakow, Poland \\ 2Department of Infectious Diseases, School of Medicine, Jagiellonian University, Krakow, Poland \\ Adv Dermatol Allergol 2018; XXXV (5): 481-484 \\ DOI: https://doi.org/10.5114/ada.2018.77238
}

\begin{abstract}
Introduction: Recent outbreaks of syphilis occurred predominantly in men who have sex with men (MSM). A significant proportion of syphilis cases occur in MSM who had more than one episode of syphilis. This group may play an important role in syphilis transmission.

Aim: To identify factors associated with the risk of syphilis reinfection.

Material and methods: Forty-four MSM patients with the first episode of syphilis who were treated at the Department of Dermatology at the Jagiellonian University School of Medicine in Krakow, Poland were included in this study. After completing the treatment, the RPR testing was done every 3 months for 2 years in every patient. In the study period, we identified $12(22 \%)$ cases of syphilis reinfection, eight of which were asymptomatic. Clinical, demographic and behavior data from patients with only one episode of syphilis were compared with those collected from repeaters.

Results: Individuals with syphilis reinfection had concomitant HIV infection more frequently, reported a higher number of sexual partners and had sex in sex on premises venues more frequently $(p<0.05)$. In the multivariate analysis, we found that being HIV-infected MSM and having sex in sex on premises venues independently correlated with a higher risk of syphilis reinfection $(\mathrm{OR}=9.6,95 \% \mathrm{Cl}: 2.2-42.5$ and $\mathrm{OR}=5.6,95 \% \mathrm{Cl}: 1.4-22.5$, respectively). Conclusions: Results of our study highlight a strong need for frequent and repeated screening among MSM patients (especially those with concomitant HIV infection) with the first episode of syphilis and taking detailed patient's history regarding also demographic and behavior data. We should also improve prevention policies to reduce risk behaviors in this population.
\end{abstract}

Key words: syphilis, HIV, sexual behavior.

\section{Introduction}

Since 2000 there has been a considerable increase in the incidence of syphilis in a number of European countries [1]. Recent studies have shown that even up to a half of episodes of syphilis can occur in people who had more than one episode of syphilis [2, 3]. It has also been suggested recently that persons with multiple syphilis reinfections can be more likely to present no symptoms. Therefore, it seems that such individuals may play a crucial role in syphilis transmission [4].

Recent outbreaks of syphilis have occurred predominantly in men who have sex with men (MSM) [3, 5] and so syphilis prevention and control efforts should be mainly focused on this core group of transmitters.
Aim

The aims of the current study were: (1) to establish the sociodemographic features of MSM with syphilis attending the Department of Dermatology at the JagielIonian University School of Medicine in Krakow, Poland, (2) to assess the incidence of syphilis reinfection among MSM, and (3) to establish factors associated with the risk of syphilis reinfection.

\section{Material and methods \\ Fofty-four consecutive patients with the first episode of early syphilis (i.e. primary or secondary) treated at the Department of Dermatology at the Jagiellonian Univer-}

Address for correspondence: Maciej Pastuszczak MD, PhD, Department of Dermatology, School of Medicine, Jagiellonian University, 8 Skawińska St, 31-066 Krakow, Poland, phone: +48 602228 796, e-mail: mpastuszczak@wp.pl Received: 2.07.2017, accepted: 27.07.2017. 
Table 1. Characteristics of patients at the time of the first syphilis episode $(n=54)$

\begin{tabular}{|c|c|}
\hline Parameter & Value \\
\hline Age (min.-max.) & $31(22-66)$ \\
\hline Men, $n(\%)$ & $54(100)$ \\
\hline \multicolumn{2}{|l|}{ Education level, $n$ (\%): } \\
\hline Primary or middle school & $2(3.7)$ \\
\hline High school or technical secondary school & $17(31.5)$ \\
\hline College degree or higher & $33(61.1)$ \\
\hline MSM, $n(\%)$ & $54(100)$ \\
\hline HIV, $n(\%)$ & $19(35.2)$ \\
\hline Reinfection of syphilis, $n$ (\%) & $12(22.2)$ \\
\hline \multicolumn{2}{|l|}{ Suspected route of acquisition of syphilis, $n$ (\%): } \\
\hline Anal intercourse & $22(40.7)$ \\
\hline Oral intercourse & $30(55.6)$ \\
\hline Vaginal intercourse & $2(3.7)$ \\
\hline Number of sexual partners in lifetime, $n$ (min.-max.) & $4(1-200)$ \\
\hline $\begin{array}{l}\text { Number of sexual partners in the past } 12 \text { months, } \\
n \text { (min.-max.) }\end{array}$ & $2(1-100)$ \\
\hline$>1$ sexual partner within last 12 months, $n(\%)$ & $31(57.4)$ \\
\hline \multicolumn{2}{|l|}{ Units of alcohol per week, $n(\%)$ : } \\
\hline 0 & $10(18.5)$ \\
\hline $1-4$ & $26(48.1)$ \\
\hline $5-10$ & $9(16.7)$ \\
\hline $11-15$ & $4(7.4)$ \\
\hline$>15$ & $5(9.3)$ \\
\hline \multicolumn{2}{|l|}{ Chemsex in the last 6 months, $n(\%)$ : } \\
\hline No & $32(59.3)$ \\
\hline One a month & $17(31.5)$ \\
\hline Once a week & $3(5.6)$ \\
\hline Almost always & $2(3.7)$ \\
\hline \multicolumn{2}{|l|}{ Sex in sex-on-premises venues, $n(\%)$ : } \\
\hline Never & $36(66.7)$ \\
\hline Once a month & $14(25.9)$ \\
\hline More than once a month, less than once a week & $3(5.6)$ \\
\hline At least once a week & $1(1.8)$ \\
\hline
\end{tabular}

Number of unprotected sexual intercourses with

unknown partners in the last 6 months, $n(\%)$ :

\begin{tabular}{lc}
\hline 0 & $19(35.2)$ \\
\hline $1-4$ & $26(48.1)$ \\
\hline $5-9$ & $2(3.7)$ \\
\hline$\geq 10$ & $5(9.3)$
\end{tabular}

Values are given as median (min.-max.), or frequencies, or otherwise stated. MSM - men who have sex with men, HIV - human immunodeficiency virus. sity School of Medicine in 2013-2016 were included in the study. Patients with latent syphilis were excluded. The diagnosis and stages of syphilis were determined by a board-certified dermatologist according to European guidelines' definitions [6]. After completing the treatment, the RPR testing was done every month in every patient until the proper serological response has been achieved (i.e. at least a 4-fold decrease in the RPR titer in comparison to the titer before treatment). Thereafter, the RPR testing was done every 3 months for 2 years or until the second episode of syphilis. Therefore, the repeat syphilis was defined as a case of syphilis occurring in a previously adequately treated patient who had a 4-fold RPR titer decrease and then experienced a 4-fold titer increase. Demographic, clinical and behavioral data were collected at the time of diagnosis of the episode of syphilis. The MSM were defined as men who reported male sex partners within the interview period (3-12 months, depending on the stage of syphilis). Sex in sex-on-premises venues was defined as a sexual intercourse with an unknown person in the places such as bathhouses, backrooms, darkrooms or gay clubs. Chemsex was defined by the use of certain drugs to facilitate or enhance sex.

All study participants gave their informed written consent, and the study was approved by the Jagiellonian University Bioethics Committee.

\section{Statistical analysis}

A statistical analysis was performed with the Statistica 7.1 PL software package (StatSoft Inc, Tulsa, OK). If not stated otherwise, data were expressed as median and minimum-maximum values. Continuous variables were compared with the Mann-Whitney $U$ test. The $\chi^{2}$ test or the Fisher exact test was used for the dichotomous variables. To identify independent factors, a multivariate logistic regression analysis was used. A p-value $<0.05$ was considered statistically significant.

\section{Results}

Fifty-four patients with primary or secondary syphilis were included in this study. All individuals were men, all were MSM and more than half had at least a college degree (Table 1). Slightly more than one-third had concomitant HIV infection. $55.6 \%$ of patients reported an oral sexual intercourse as a presumptive route of syphilis transmission. Almost $60 \%$ of individuals reported having more than one sexual partner within the last 12 months prior to the diagnosis of syphilis. $40.8 \%$ of patients reported having chemsex at least once a month within the last 6 months prior the diagnosis. One-third of individuals had regular sex in sex-on-premises venues (i.e. at least once a month).

Syphilis was diagnosed more than once in 12 (22\%) patients. Eight of 12 patients with repeat syphilis were asymptomatic, two had chancre and the remaining two 
were diagnosed as having secondary syphilis. The mean time from the first episode of syphilis to the reinfection was 12 months (min.-max.: 6-24 months).

Patients with syphilis reinfection when compared to those with the single episode of the disease did not differ with respect to the education level, route of syphilis transmission and behavior such as alcohol consumption and frequency of using drugs to facilitate or enhance sex (chemsex) (Table 2). However, individuals with a repeat syphilis infection were significantly older, had HIV infection more frequently and reported a higher number of sexual partners in their lifetime $(p<0.05)$. Patients with the first episode of syphilis reported having sex in sexon-premises venues less frequently than patients with syphilis reinfection $(p<0.05)$ (Table 2 ).

In the multivariate analysis, syphilis reinfection was strongly associated with being HIV-infected (OR = 9.6; 95\% Cl: 2.2-42.5) and having sex in sex-on-premises venues $(\mathrm{OR}=5.6 ; 95 \% \mathrm{Cl}: 1.4-22.5)\left(R^{2}=0.29 ; p=0.0009\right)$.

\section{Discussion}

Sexually transmitted infection (STI) prevention and control efforts, including those for syphilis, have traditionally focused on the core groups of transmitters. Since 2000 syphilis incidence among MSM has been on a global rise. Further studies showed that the majority of episodes of syphilis occurred in MSM who had at least one previous episode of syphilis [1, 2]. Moreover, it has been observed that persons with repeat syphilis were more likely to present with asymptomatic disease [3, 4]. Therefore, such patients are pointed out as an important core group in the genesis of current syphilis outbreaks.

In this study, the proportion of repeat syphilis cases among MSM was compared with MSM who had only one episode. Our aim was to investigate factors potentially associated with the repeat infection.

The incidence of repeat syphilis in our study was $22 \%$. However, it varies between studies and ranges between approximately 10\% in US studies and up to $40 \%$ in European ones [1-3]. It can be partially explained by different definitions of syphilis reinfection used in studies. Another explanation can be a different approach to patients after syphilis treatment. In some countries, after completing the treatment and proper clinical and serological response (i.e. at least a 4-fold decrease in RPR titer), patients are asked to repeat serological testing every 3-6 months. Such approach seems to detect reinfections more effectively.

In the current study we found two independent factors associated with syphilis reinfection. HIV-infected MSM had a nearly ten times greater risk of repeat syphi-

Table 2. Comparison of patients with a single episode of syphilis and syphilis reinfection

\begin{tabular}{|c|c|c|c|}
\hline Parameter & Reinfection of syphilis $(n=12)$ & Single episode of syphilis $(n=42)$ & $P$-value \\
\hline Age (min.-max,) & $38(22-66)$ & $30(22-65)$ & 0.04 \\
\hline MSM, $n(\%)$ & $12(100)$ & $38(90.5)$ & NS \\
\hline HIV, $n(\%)$ & $9(75)$ & $10(23.8)$ & 0.002 \\
\hline \multicolumn{4}{|l|}{ Education level, $n(\%)$ : } \\
\hline Primary or middle school & $0(0)$ & $2(4.8)$ & NS \\
\hline High school or technical secondary school & $2(16.7)$ & $16(38.1)$ & NS \\
\hline College degree or higher & $9(75)$ & $24(57)$ & NS \\
\hline \multicolumn{4}{|l|}{ Route of syphilis transmission, $n$ (\%): } \\
\hline Anal intercourse & $3(25)$ & $18(42.9)$ & NS \\
\hline Oral intercourse & $8(66.7)$ & $18(42.9)$ & NS \\
\hline Vaginal intercourse & $0(0)$ & $4(9.5)$ & NS \\
\hline $\begin{array}{l}\text { Number of sexual partners in lifetime } \\
\text { (min.-max.) }\end{array}$ & $8.5(3-200)$ & $3(1-200)$ & 0.02 \\
\hline $\begin{array}{l}\text { Number of sexual partners in the last } 12 \text { months } \\
\text { (min.-max.) }\end{array}$ & $4(1-100)$ & $2(1-20)$ & 0.02 \\
\hline$>1$ sexual partner within last 12 months, $n(\%)$ & $10(83.3)$ & $21(50)$ & 0.04 \\
\hline $\begin{array}{l}\text { At least } 1 \text { unprotected sexual intercourse with } \\
\text { unknown partner within last } 12 \text { months, } n(\%)\end{array}$ & $10(83.3)$ & $21(50)$ & 0.04 \\
\hline More than 10 alcohol units per week, $n$ (\%) & $2(16.7)$ & $8(19)$ & NS \\
\hline Never Chemsex, $n(\%)$ & $6(50)$ & $26(61.9)$ & NS \\
\hline Never sex in sex-on-premises venues, $n(\%)$ & $4(33.3)$ & $31(73.8)$ & 0.03 \\
\hline
\end{tabular}


lis compared with HIV-uninfected MSM. All of HIV-infected individuals were on antiretroviral therapy. Such observations are consistent with those of previous studies [4, 7-9]. The link between repeat syphilis and HIV infection might result from specific sexual behaviors such as serosorting (the practice of seeking sex partners of the same HIV status) and reduced condom use. On the other hand, HIV-infected persons receive HIV care and routine screening for STIs. It can contribute to increased case detection of syphilis reinfection.

Secondly, we found for the first time that sex in sex-on-premises venues is an independent risk factor for syphilis reinfection. More than two-thirds of individuals with repeat syphilis compared with a quarter of those with the first episode reported at least one sexual intercourse in places such as sex clubs within the last 2 years. Interestingly, $78 \%$ of all patients visiting sex clubs reported having only unprotected oral intercourses.

While oral sex is perceived as a low risk factor for the acquisition of HIV, it is considered as a high risk factor for the transmission of syphilis as oral lesions are highly infectious. Therefore, in the current study we found that more than a half of individuals stated oral sex as the only risk factor for the acquisition of infection. Our findings are similar to those derived from the study of Hourihan et al. [9].

Previous studies showed that patients with repeat syphilis were more likely to present with asymptomatic disease [3, 4]. In the current study, we confirmed these findings showing that almost $70 \%$ of repeaters were diagnosed at a latent stage of syphilis. We presume that it can be related to the regular and frequent screening for syphilis (i.e. every three months according to the study protocol). However, some authors speculate that the higher number of asymptomatic cases of repeat syphilis can be associated with T. pallidum-induced partial immunity to reinfection [10].

The most important limitation of our study is a small sample size. Therefore, the results cannot be extrapolated directly on the whole MSM population. Despite the increasing incidence of the syphilis rate, the disease is quite rare with the mean frequency in Poland up to 5 cases per 100,000 people. On the other hand, the strength of this study is the prospective cohort of patients. We have observed the participants since the first episode of syphilis. There were no missing data as in retrospective studies. All repeated diagnostic tests (such as RPR) were always performed in the same laboratory using the same methods. Moreover, it was also possible to use rigorous definitions of repeat episode.

Despite these limitations, this study provides some important findings in the field of repeat syphilis in the European population. Syphilis elimination efforts should include messages about the risk of repeat syphilis infection. Our data suggest that HIV infection and behavioral factors such as having sex in sex-on-premises venues are important determinants of the risk of syphilis reinfection among MSM. Therefore, it seems that frequent and repeated syphilis testing after the first syphilis infection may help reduce rates of syphilis. Results of studies such as ours reiterate the need for more innovative approaches to reduce risk behaviors among MSM population.

\section{Conflict of interest}

The authors declare no conflict of interest.

\section{References}

1. European Centre for Disease Prevention and Control (ECDC). Sexually transmitted infections in Europe 2011. Stockholm: ECDC; 2013. Available from: http://ecdc.europa.eu/en/ publications/Publications/sexually-transmitted-infectionseurope-surveillance-report-2012.pdf.

2. Kenyon C, Lynen L, Florence E, et al. Syphilis reinfections pose problems for syphilis diagnosis in Antwerp, Belgium 1992 to 2012. Euro Surveill 2014; 19: pii20958.

3. Katz KA, Lee MA, Gray T, et al. Repeat syphilis among men who have sex with men - San Diego County, 2004-2009. Sex Transm Dis 2011; 38: 349-52.

4. Brewer TH, Peterman TA, Newman DR, et al. Reinfections during the Florida syphilis epidemic, 2000-2008. Sex Transm Dis 2011; 38: 12-7.

5. Abara WE, Hess KL, Neblett Fanfair R, et al. Syphilis trends among men who have sex with men in the United States and Western Europe: a systematic review of trend studies published between 2004 and 2015. PLoS One 2016; 11: e0159309.

6. Pastuszczak M, Wojas-Pelc A. Current standards for diagnosis and treatment of syphilis: selection some practical issues, based on the European (IUSTI) and U.S. (CDC) guidelines. Postep Dermatol Alergol 2013; 30: 203-10.

7. Phipps W, Kent C, Kohn R, et al. Risk factors for repeat syphilis in men who have sex with men. San Francisco. Sex Transm Dis 2009; 36: 331-5.

8. Cohen SE, Chew Ng RA, Katz KA, et al. Repeat syphilis among men who have sex with men in California, 2002-2006: implications for syphilis elimination efforts. Am J Public Health 2012; 102: e1-8.

9. Hourihan $\mathrm{M}$, Wheeler $\mathrm{H}$, Houghton R, et al. Lessons from the syphilis outbreak in homosexual men in east London. Sex Transm Infect 2004; 80: 509-11.

10. Morgan CA, Lukehart SA, Van Voorhis WC. Protection against syphilis correlates with specificity of antibodies to the variable regions of Treponema pallidum repeat protein $\mathrm{K}$. Infect Immun 2003; 71: 5605-12. 